Research Paper:

\title{
Quality of Life and Some Related Factors in Patients crossuart With Beta Thalassemia Major in Southwest Iran
}

Mostafa Madmoli ${ }^{1}$, Yaghoob Madmoli ${ }^{2}$, Peyman Rahmati ${ }^{1}$, Ahmad Adavi $^{3^{*}}$, Najme Yousefi ${ }^{4}$ Zohre Gheisari $^{5}$, Marzie Abbaszade Aliabad ${ }^{6}$

1. Student Research Committee, Dezful University of Medical Sciences, Dezful, Iran.

2. Student Research Committee, Ahvaz Jundishapur University of Medical Sciences, Ahvaz, Iran.

3. Department of Nursing, Faculty of Nursing and Midwifery, Dezful University of Medical Sciences, Dezful, Iran.

4. Imam Ali Hospital, Gerash University of Medical Sciences, Gerash, Iran.

5. Student Research Committee, Shushtar Faculty of Medical Sciences, Ahvaz Jundishapur University of Medical Sciences, Ahvaz, Iran

6. Student Research Committee, Kerman University of Medical Sciences, Kerman, Iran.

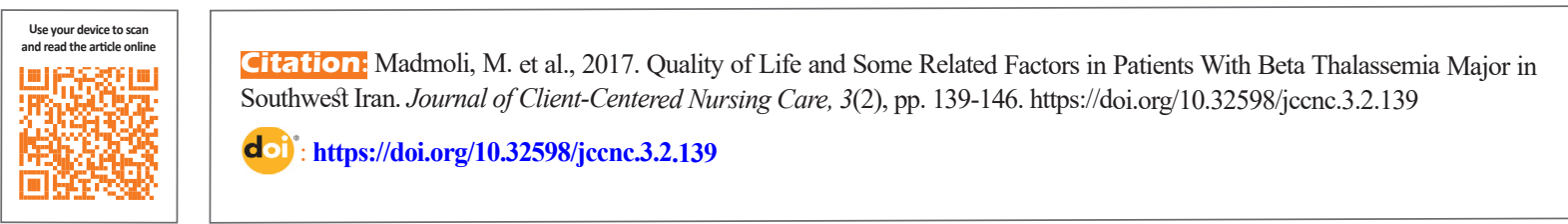

Article info:

Received: 03 Jan. 2017

Accepted: 29 Mar. 2017

Keywords:

Beta thalassemia, Nurses, Health related quality of life

\begin{abstract}
A B S T R A C T
Background: Thalassemia is one of the most common genetic disorders in the world, especially in Iran. The study of various aspects affecting the physical and mental health of patients with beta thalassemia major has become more important. The aim of this study was to evaluate the Quality of life and some related factors in patients with beta-thalassemia major in Southwest Iran.

Methods: In this cross-sectional, descriptive study, quality of life and related factors in 112 thalassemia major patients with available sampling attending Dezful Thalassemia Center has been studied in the first three months of 2015. Data collection was based on self-report including demographic questionnaire and 36-SF quality of life questionnaire. Finally, the data were analyzed using SPSS software version 18 and chi-square test, T-test, and Pearson's correlation coefficients.

Results: Patients with beta thalassemia major, including 66 males $(58.92 \%)$ and 46 females $(41.07 \%)$ with a mean age of 23 years were enrolled in the study. The lowest and highest scores were obtained in general health (63.59) and physical functioning (85.25), respectively. Findings showed that there is no statistically significant difference $(\mathrm{P}=0.025)$ between men and women in all aspects of quality of life except role disorder. It was also found that married people have a higher quality of life and mental health in comparison to other people $(\mathrm{P}<0.05)$. In addition, there was a negative correlation between age and physical health, mental health, and quality of life total scores which were not statistically significant $(\mathrm{P}=0.46)$.

Conclusion: The present study results showed well that age, income level, and marital status indices play a very important role in the maintenance of quality of life and the reduction of negative effects of disease on patient's mental and physical health. The limitations of this study are to examine the dimensions of quality of life in a center. It seems that future studies should look at ways to improve their quality of life.
\end{abstract}

\section{* Corresponding Author:}




\section{Background}

halassemia is a group of hereditary blood disorders caused by defects in the synthesis of one or more hemoglobin chains (Muncie \& Campbell 2009). The disease is a serious public health problem throughout the Mediterranean $\mathrm{T}$ region, the Middle East, the Indian subcontinent, and
Southeast Asia (Thavorncharoensap et al. 2010). There are more than two million carriers of beta thalassemia and more than 15 thousand beta thalassemia major patients in Iran. The disease is more or less found to be common in the Northern provinces and Southern shores of Iran (Azad, Shiargar \& Kazemi Haki 2015). Patients with thalassemia are marked with symptoms such as chronic and severe anemia, lack of growth, enlargement of the spleen and liver, bone disorders, especially visible changes in the craniofacial skeleton, and disguise (Khani et al. 2012).

Treatment is based on frequent blood transfusions and iron chelation drugs. In spite of the treatment measures that increase the survival of these patients, its clinical signs adversely affect the physical and mental health of the patients and their family. These physical and psychological problems lead to disappointment, decreased function, and ultimately reduced the quality of life (Arian et al. 2013).

Unfortunately, in recent years, in spite of developments in medical treatment and increase in survival rate, experts have paid less attention to the social and mental aspects of the disease. Therefore, helping these patients is mainly limited to basic medical services to sustain life (Zare et al. 2014). Factors such as course duration of disease and treatment, hospitalization and increased medical costs, mental status, and social problems cause stress in patients with chronic disease and their family (Alavi et al. 2007).

A chronic disease always leads to limitations in quality of life, particularly if repeated and complex treatments were needed. Studies have shown that thalassemia major and even intermediate thalassemia that are not related to injection greatly reduce the quality of life of patients (Hamed Tavassoli \& Alhani 2012). The primary purpose of most of these patients was to improve the quality of life by reducing the effects of the disease, and they do not necessarily have a low quality of life (Alijany Renany et al. 2012). Since the quality of life includes a range of human objectives and needs, which can be achieved in association with individual and group with the understanding of feeling of well-being, knowing to what extent and in what aspects the patients feel good or do not, it helps to plan in such a way as to lessen their suffering (Hadi, Karami \& Montazeri 2009).

Different studies have shown that patients with thalassemia had a low quality of life, especially in psychological and emotional dimensions of quality of life as compared to their peers. Khani et al., showed that $64.9 \%$ of thalassemia patients lacked healthy mental health and $20.5 \%$ were suspected of mental illness (Khani et al. 2012). In the study conducted by Khalidi et al., it was found that the main problem the children and adolescents with thalassemia dealt with was emotional dimension of the disease and thus, had the lowest mean score of quality of life in emotional function domains (Khaledi, Moridi \& Valiee 2013). Impairment of quality of life imposed a negative impact on social life, family, work, and recreational activity and increased the risk of hospitalization and death from this disease (Moser 2002).

According to the reviews, few studies have been done in Khuzestan, one of the five provinces with the highest prevalence of thalassemia in Iran (Zare et al. 2014). Since designing a proper plan for promoting patient's health level requires sufficient knowledge about the different dimensions of quality of life, researchers had prompted a study to assess the Quality of life and some related factors in patients with betathalassemia major in Southwest Iran in 2015.

\section{Materials \& Methods}

\section{Design and sample}

The present study is a cross-sectional, descriptive, which was done after approval and permission from the officials of Dezful University of Medical Sciences (DUMS-121) and after obtaining the informed consents of patients in the thalassemia ward of Dezful large hospital. The study was performed in available sampling method based on inclusion and exclusion criteria. The total number of patients with thalassemia in the center was 158, out of which 112 patients were included the study in 2015. Inclusion criteria for this study were: thalassemia major, aged between 15 and 25 years, literate, having at least one hospitalization record, not having chronic diseases or severe physical complications caused by the disease, and able to communicate and complete the questionnaire independently. 


\section{Data collection}

In the present study, self-report based data collection method was employed; therefore demographic characteristic form and SF-36 quality of life questionnaire were used as data collecting tools. Demographic characteristic form includes age, gender, Body Mass Index (BMI), race, education level, occupation, marital status, monthly income, hospitalization record, and medications. The SF-36 questionnaire was used to investigate the patients' quality of life. The questions include eight concepts: physical function, social function, role disorder due to physical health, pain, general health, role disorder due to emotional health, energy/fatigue, and emotional well-being domains. Each question had a maximum score of 100 and a minimum of zero.

Also there are 2 questions with scores 50 and 100; 3 questions with scores of 0,50 and 100; 5 questions with scores of $0,25,50,75$ and 100; 6 questions with scores of $0,20,40,60,80$ and 100 were considered. Physical health score is calculated by averaging total score of physical function, role disorder due to physical health, pain, and general health domains. The average of total scores of all the eight concepts will be calculated to measure the total score of quality of life. The average of each concept was calculated for each individual. A maximum of 100 and a minimum of 0 is considered for each dimension and total score of quality of life. A score less than 50 means low quality of life and more than 50 means high quality.

The SF-36 questionnaire is a standard criterion which is considered to be used in research and clinical practice, hygiene policy evaluation, health, and health status checking of general population. Using Cronbach's alpha test, in 1992 in the UK, Brazier et al., determined its reliability above $85 \%$ (Mahmoudi et al. 2012). In a study conducted by Montazeri et al., in Iran with the aim of translation, reliability, and validity determination of Persian version of SF-36 questionnaire showed that except for the vitality scale $(\alpha=0.65)$, other scales of Persian version of SF-36 had the minimum standard of reliability coefficients in the range of 0.77 to 0.9 (Montazeri, Goshtasbi \& Vahdaninia 2006). The validity of this questionnaire was determined by formal method, and its reliability was 0.78 using Cronbach's alpha test. After the initial selection of samples and their informed consent, the SF-36 questionnaire of quality of life was given to them.

\section{Statistical analysis}

The data were analyzed using SPSS software version 18. A P-value of less than 0.05 was considered as sig- nificant level. Kolmogorov-Smirnov test showed that variables distribution of quality of life, physical health, and mental health was normal. Therefore, parametrical statistic test was used. No statistically significant difference was observed between men and women in all dimensions of quality of life except role disorder due to physical health $(\mathrm{P}=0.025)$ (Figure 1). Compared to other patients, married people had a higher quality of life and mental health, which was a statistically significant difference $(\mathrm{P}<0.05)$. No significant statistical relationship was observed among other indicators and variables of quality of life $(\mathrm{P}>0.05)$ (Table 1).

There was a direct correlation between income and body weight with physical health, mental health and quality of life scores but it was not statistically significant $(\mathrm{P}>$ 0.05) (Figure 2). Also, there was a negative correlation between age and physical health, as well as metal health and quality of life total scores which was not statistically observed significant $(\mathrm{P}=0.46)$ (Figure 3 ).

\section{Results}

66 males (58.92\%) and 46 females (41.07\%) with thalassemia with an average age of 23 (5.4) years were enrolled. Other demographic variables are listed in Table 1. The mean score of quality of life was 29.72 (19.17). The lowest and highest scores were obtained in general health (63.59), and physical functioning (85.25), respectively. Patients' group physical health mean was in an average level of 5.19 scores higher than their mental health mean. There was a significant relationship between quality of life and marital status $(\mathrm{P}=0.02)$ (Table 2).

No statistically significant difference was observed between men and women in all dimensions of quality of life except role disorder due to physical health ( $\mathrm{P}=$ 0.025) (Figure 1). Compared to other patients, married people had a higher quality of life and mental health, which had a statistically significant difference $(\mathrm{P}<0.05)$. No significant statistical relationship was observed among other indicators and variables of quality of life (P $>0.05$ ) (Table 1).

There was a direct correlation between income and body weight with physical health, mental health and quality of life scores but it was not statistically significant $(\mathrm{P}>0.05)(\mathrm{r}=0.23)$ (Figure 2$)$. Also, there was a negative correlation between age and physical health, as well as for mental health and quality of life total scores, which were observed to be statistically insignificant $(\mathrm{P}=$ 0.46) $(\mathrm{r}=0.15)$ (Figure 3). 
Table 1. Correlation of quality of life and its dimensions with demographic variables of patients with thalassemia major in Dezful, 2016 using descriptive statistics

\begin{tabular}{|c|c|c|c|c|c|}
\hline \multirow{2}{*}{ Variable } & & \multirow{2}{*}{ No. (\%) } & \multirow{2}{*}{$\begin{array}{c}\text { Quality of Life } \\
\text { Test Results }\end{array}$} & \multirow{2}{*}{$\begin{array}{c}\text { Physical Health } \\
\text { Test Results }\end{array}$} & \multirow{2}{*}{$\begin{array}{c}\text { Mental Health } \\
\text { Test Results }\end{array}$} \\
\hline & & & & & \\
\hline \multirow[b]{2}{*}{ Gender } & Male & 66 (58.92) & \multirow[b]{2}{*}{0.06} & \multirow[b]{2}{*}{0.57} & \multirow[b]{2}{*}{0.82} \\
\hline & Female & $46(41.07)$ & & & \\
\hline \multirow[b]{2}{*}{ Marital status } & Single & $93(83.00)$ & \multirow[b]{2}{*}{$0.02^{*}$} & \multirow[b]{2}{*}{0.07} & \multirow[b]{2}{*}{$0.03^{*}$} \\
\hline & Married & $19(17.00)$ & & & \\
\hline \multirow{3}{*}{ Location } & City & $73(65.2)$ & \multirow{3}{*}{0.28} & \multirow{3}{*}{0.16} & \multirow{3}{*}{0.38} \\
\hline & & & & & \\
\hline & Rural & $39(34.8)$ & & & \\
\hline \multirow{5}{*}{ Education } & Illiterate & $4(3.6)$ & \multirow{5}{*}{0.34} & \multirow{5}{*}{0.41} & \multirow{5}{*}{0.27} \\
\hline & Under high school & $48(42.9)$ & & & \\
\hline & High school & $41(36.6)$ & & & \\
\hline & Diploma degree & $3(2.7)$ & & & \\
\hline & University degree & $16(14.3)$ & & & \\
\hline \multirow{3}{*}{ Hospitalization history } & Yes & $67(59.8)$ & \multirow{3}{*}{0.51} & \multirow{3}{*}{0.42} & \multirow{3}{*}{0.34} \\
\hline & & & & & \\
\hline & No & $45(40.2)$ & & & \\
\hline \multirow{3}{*}{ Surgery history } & Yes & $41(36.6)$ & \multirow{3}{*}{0.34} & \multirow{3}{*}{0.11} & \multirow{3}{*}{0.31} \\
\hline & & & & & \\
\hline & No & $71(63.4)$ & & & \\
\hline \multirow{9}{*}{ Blood group } & $\mathrm{A}^{+}$ & $39(34.8)$ & \multirow{9}{*}{$0.02^{*}$} & \multirow{9}{*}{$0.03^{*}$} & \\
\hline & $A^{-}$ & $1(0.9)$ & & & \\
\hline & $\mathrm{B}^{+}$ & $25(22.3)$ & & & \\
\hline & $\mathrm{B}^{-}$ & $1(0.9)$ & & & \\
\hline & & & & & $0.01^{*}$ \\
\hline & $\mathrm{AB}^{+}$ & $7(6.3)$ & & & \\
\hline & $A B$ & $0(0)$ & & & \\
\hline & $\mathrm{O}^{+}$ & $39(34.8)$ & & & \\
\hline & $\mathrm{O}^{-}$ & $0(0)$ & & & \\
\hline
\end{tabular}

* Significant level

Client-Centered Nursing Care 


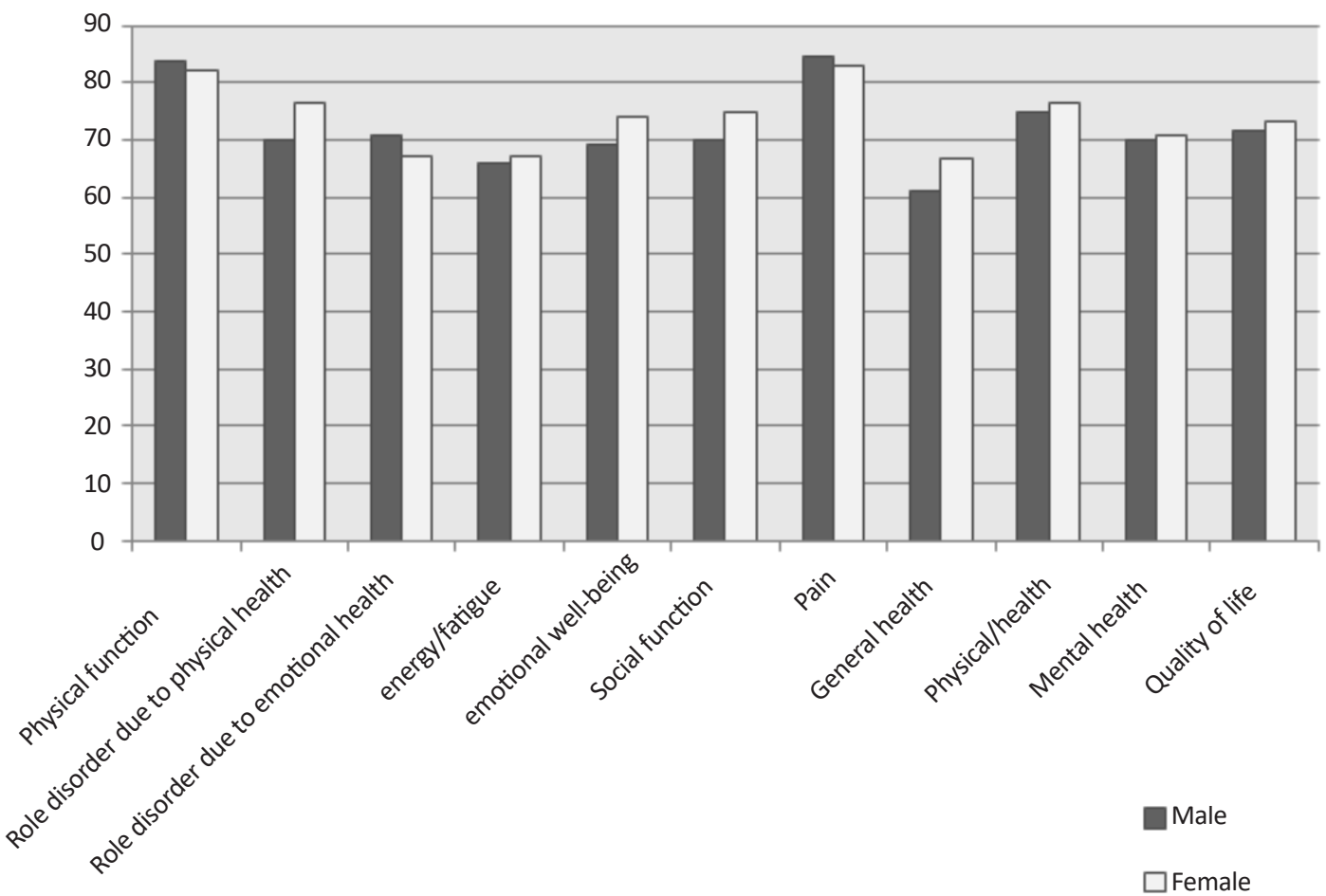

Client-Centered Nursing Care

Figure 1. Comparison of the mean scores of the eight dimensions of quality of life related to health, physical health, mental health and quality of life total scores for men and women suffering from thalassemia major

\section{Discussion}

The aim of this study was to assess the quality of life and some related factors in patients with beta-thalassemia major in southwest Iran in 2015. Results showed that there were significant relationships among physical and mental health dimensions and factors affecting their qualities in relation to aspects of physical and mental health and related factors in patients with beta-thalassemia. The significant effect of patients' marital status on quality of life dimensions was a considerable result of the study. In other words, since married people receive

Table 2. The mean and standard deviation of 8 dimensions of quality of life related to health, physical health, mental health and quality of life total scores in patients with beta thalassemia major using descriptive statistics

\begin{tabular}{cl}
\hline Health Dimensions & Mean (SD) \\
\hline Physical function & $86.25(17.49)$ \\
\hline Role disorder due to physical health & $72.54(32.35)$ \\
Role disorder due to emotional health & $69.32(34.70)$ \\
Energy / fatigue & $66.42(23.32)$ \\
Emotional well-being & $71.30(20.56)$ \\
Social function & $72.18(23.27)$ \\
Pain & $84.06(19.62)$ \\
General health & $63.59(19.86)$ \\
Mensical health & $75.58(16.11)$ \\
\hline Quality of life total score & $70.39(20.30)$ \\
\hline
\end{tabular}




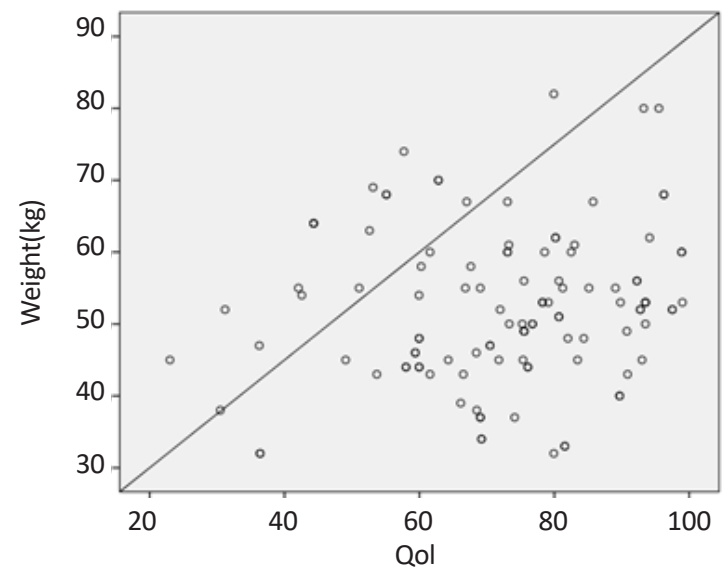

Client-Centered Nursing Care

Figure 2. Correlation between weight and quality of life total score in patients with beta thalassemia major

mental patronage as well as physical and medical care from their families, therefore they were imposed to less physical and mental damages compared to single ones. The patients' self-support can stimulate their forgotten abilities and apply them to eliminate the defects imposed on them by thalassemia and use it as a positive point to reinforce their ambitions and self-confidence. Gollo et al. (2013) showed that there was no difference but the findings of the present study were in contrary to it and a little predominance was observed in males.

Findings of the present study had a correlation with the findings of Hadi et al. (2009). In a study "quality of life related to health in patients with thalassemia major", they showed that the level of quality of life in patients with thalassemia with a lower score in physical health and its related dimensions was lower compared to that of control group.

Also, results showed that since educated patients enjoyed a better understanding, they had a higher level of quality of life as well as physical and mental health and therefore, they did better in dealing with the negative effects of the disease and showed a higher level of selfcare. By taking the responsibility of self-care, they were able to recover from defects and inabilities resulting from the disease and also applied self-care potential to solve their problems, but this was not statistically significant. The present study results correlate to Masoudi et al., (2009) and Azad et al., (2015) in the mean score of quality of life mental dimensions for role play limitations.

Results showed that in all quality of life dimensions except role disorder, there was no statistically significant difference between men and women due to physical

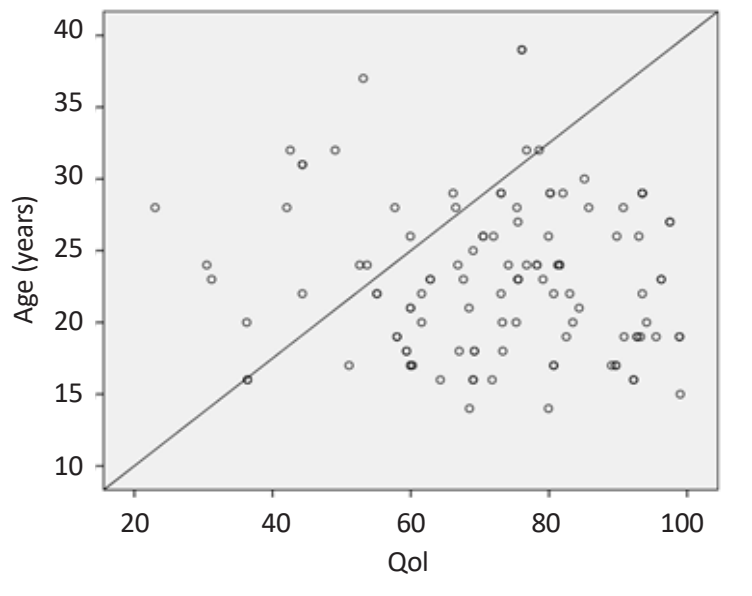

Client-Centered Nursing Care

Figure 3. Correlation between age and quality of life total score in patients with beta thalassemia major

health. Married patients had a higher level of quality of life and mental health. In other words, married patients' emotional health, energy/fatigue, emotional well-being, and social function faced less damage in comparison to single ones, which was statistically significant. No statistically significant relationship was observed in between other indices (blood group, education, hospitalization and/or surgery, and gender) and quality of life variables. Also, there was a negative correlation between age and physical health, as well as mental health and quality of life total scores, which was not statistically significant.

The negative effect of thalassemia on social activity restriction considerably increases the fear of banishment, endangering the independence, and future anxiety in patients. The life for a patient with thalassemia means repeated story of the pains of the individual who has to receive blood monthly, sleep with desferal pumps, and feel the sting of injections in every inch of the body. Thinking of these difficulties will, without a doubt, make even a healthy person face severe, unimaginable mental trauma. Therefore, it is beyond imagination how a patient with thalassemia engages in these problems, which naturally affects the mental health of the patient. Hence, these problems lead to the degradation of the quality of life of the patient.

It is evident that a patient with thalassemia needs continuous care to reduce the complications and help them enjoy an active and normal life. Since, the aforementioned difficulties of the disease have a negative effect on the patient's mental and physical health and the quality of life, the role of parents, spouse, and even children in providing mental and medical support is very important and requires serious cooperation of health organizations 
for prompt prevention and/or remedial support. Definitely, the quality of services will have a great effect on the patient's mental and physical health levels and consequently, on their quality of life.

There was a direct correlation between income and physical health, mental health, and the patients' quality of life which was not statistically significant. The quality of remedial services accessed including special remedial cares, blood, and required medicines play a decisive role in the patients' quality of life, which has a direct relationship to their financial ability. In fact, those with suitable financial level showed a higher level of self-confidence and life expectation compared to those with a lower financial level who had to wait for a longer time for remedial process. This finding correlates with the findings of Tahmasbi et al., (2007).

As for the findings of the present study, it is obvious that patients with thalassemia need special attention from family and friends for self-care, physical (medical) care, and mental support, as well as from government for remedial costs. Therefore, it is recommended to provide health and remedial centers with required facilities regarding feasible access to blood and medicines, as well as employment of knowledgeable personnel with enough skills in communicating with these patients to improve and maintain the quality of life. Additionally, emphasis must be on the role of family and informing those with such patients as to how support and care should be taken.

In general, the present study results showed the quality of life of these people is at a moderate level. Also age, income level, and marital status indices play a very important role in the maintenance of quality of life and the reduction of negative effects of the disease on the patient's mental and physical health. Comparison of various dimensions of quality of life between men and woman showed that both genders are equally influenced by all dimensions of quality of life (physical function, pain, general health, emotional health, energy/fatigue, emotional well-being, social function) except role disorder.

The following recommendations can be useful: Increasing the patients' capacitation by presenting selfcare and self-management educations to the patients and their families; and Encouraging the patients to maintain physical and mental health by doing exercise and attending training workshops and meetings, as well as receiving required training by remedial cadre and as a result, enabling the patients in self-care.
The limitations of this study are to examine the dimensions of quality of life in a center and not to consider some factors such as insurance coverage of affected people and also the absence of some of them from the thalassemia center to continue the remedial procedure. It seems that future studies should look at ways to improve the quality of life of the patients.

\section{Acknowledgments}

This study has been conducted with financial support from Deputy of Research of Dezful University of Medical Sciences and its ethical code is DUMS-121 from research proposal.

\section{Conflict of Interest}

The authors declare that there are no conflicts of interest.

\section{References}

Alavi, A., et al., 2007. [Comparison of perspective of children with major thalassemia and their parents about their quality of life in Shahrekord (Persian)]. Journal of Shahrekord University of Medical Sciences, 8(4), pp. 35-41.

Alijany Renany, H., et al., 2012. [The effect of using partnership care model on the quality of life in the school age children with $\beta$-thalassemia (Persian)]. Journal of Shahrekord University of Medical Sciences, 14(1), pp. 41-9.

Arian, M., et al., 2013. [Impact of an 8-week walking program on quality of life in patients with thalassemia major (Persian)] KAUMS Journal (FEYZ), 17(5), pp. 463-70.

Azad, M., Shiargar, P. \& Kazemi Haki, B., 2015. [Quality of life in patients with thalassemia major referred to Ardabil Buali Hospital in 2012-13 (Persian)]. Medical Science Journal of Islamic Azad Univesity-Tehran Medical Branch, 25(4), pp. 305-10.

Costantini, M. et al., 2013. Changes in the quality of life of people with thalassemia major between 2001 and 2009. Patient Preference and Adherence, 7, pp. 231-6. doi: 10.2147/ppa.s42133

Gollo, G., et al., 2013. Changes in the quality of life of people with thalassemia major between 2001 and 2009. Patient Preference and Adherence, 7, pp. 231-6. doi: 10.2147/PPA.S42133

Hadi, N., Karami, D. \& Montazeri, A., 2009. [Health related quality of life in major thalassemic patients (Persian)]. Payesh Journal, 8(4), pp. 387-93.

Hamed Tavassoli, S. \& Alhani, F., 2012. [Nurses' problems in promoting quality of life of children suffering from thalassemia (Persian)]. Preventive Care in Nursing \& Midwifery Journal, 2(1) pp. 36-44. 
Khaledi, S. H., Moridi, G. \& Valiee, S., 2013. [Comparison the quality of life of healthy and thalassemic children (Persian)]. Iranian Journal of Nursing Research, 8(2), pp. 87-94.

Khani, H., et al., 2012. Quality of life of Iranian [Beta]-thalassaemia major patients living on the southern coast of the Caspian Sea. Eastern Mediterranean Health Journal, 18(5), pp. 539-45. PMID: 22764445

Mahmoudi, G. R., et al., 2012. [Effect of self care program on the quality of life in sickle cell anemia (Persian)]. Jundishapoor Scientific Medical Journal, 11(2), pp. 201-11.

Masoudi, R., et al., 2009. [The effect of self care program education based on Orem's theory on mental aspect of quality of life in multiple sclerosis patients (Persian)]. Iran Journal of Nursing, 22(60), pp. 53-64.

Montazeri, A., Goshtasbi, A. \& Vahdaninia, M. A. S., 2006. [The short form health survey (SF-36): Translation and validation study of the Iranian version (Persian)]. Payesh, 5(1), pp. 49-56.

Moser, D. K., 2002. Psychosocial factors and their association with clinical outcomes in patients with heart failure: Why clinicians do not seem to care. European Journal of Cardiovascular Nursing, 1(3), pp. 183-8. doi: 10.1016/s1474-5151(02)00033-6

Muncie Jr. H. L. \& Campbell, J., 2009. Alpha and beta thalassemia. American Family Physician, 80(4), pp. 339-44. PMID: 19678601

Tahmasbi, S., Aein, F. \& Heravi Karimuoe, F., 2007. B-thalassemia children and quality of life: Does the present condition justify children's quality of life. Journal of Holistic Nursing And Midwifery, 17(1), pp. 23-30.

Thavorncharoensap, M., et al., 2010. Factors affecting healthrelated quality of life in Thai children with thalassemia. BMC Hematology, 10(1). doi: 10.1186/1471-2326-10-1

Zare, K., et al., 2014. [Comparison of quality of life of young people with thalassemia referred to thalassemia Shafa hospital in Ahvaz members of their families (Persian)]. Research in Nursing, 8(4), pp. $42-50$. 